\title{
Characterization of the PEG layer of sterically stabilized liposomes: a SAXS study
}

\author{
Z. Varga ${ }^{1}$, A. Wacha ${ }^{1}$, U. Vainio ${ }^{2}$, J. Gummel ${ }^{3}$, A. Bóta ${ }^{1}$ \\ ${ }^{1}$ Department of Biological Nanochemistry, Institute of Nanochemistry and Catalysis, Chemical \\ Research Center, Hungarian Academy of Sciences, Pusztaszeri út 59-67, H-1025, Budapest, Hungary \\ ${ }^{2}$ Hamburger Synchrotronstrahlungslabor, HASYLAB at Deutsches Elektronen-Synchrotron DESY, \\ Notkestrasse 85, 22607 Hamburg, Germany \\ ${ }^{3}$ European Synchrotron Radiation Facility, ESRF, 6 Rue Jules Horowitz, BP 220, 38043 Grenoble, \\ Cedex 9, France
}

Abstract

Synchrotron small-angle X-ray scattering analysis of the bilayer structure of a pharmacologically relevant sterically stabilized liposome system is presented. Describing the electron density profile of the bilayer with the superposition of Gaussian functions, the contribution of the poly(ethylene glycol) (PEG) layers to the total electron density was identified. The changes in the thickness of the PEG layer as well as the distribution of the PEG chains among the outer and inner leaflets of the bilayers were followed by changing the molar ratio of the PEG-lipid and the molar weight of the PEG molecule.

\section{Keywords}

liposomes; structural characterization; small-angle X-ray scattering; poly(ethylene glycol); sterical stabilization

\section{Introduction}

The appearance of liposomal drug delivery systems was the beginning of the continuously growing field of nanomedicine. Though the idea of using liposomes as carriers for different drugs came just a few years after the observation of the formation of spherical lamellar structures from phospholipids in excess water (Bangham, 1995), it took several decades until liposomal formulations reached the pharmaceutical product level (Barenholz, 2001). An important breakthrough on this field was the development of long-circulating liposomes (also called as sterically stabilized liposomes, SSLs), which was achieved by the incorporation of lipopolymers into the liposomal constituents. The ubiquitous lipopolymer in SSLs is polyethylene glycol (PEG) covalently attached to phosphoethanolamine (mPEGDSPE or DSPE-PEG), which assign the 'stealth' character to these drug delivery vehicles (Barenholz, 2001 and citations therein). The first liposomal drug was Doxil, a PEGylated liposomal doxorubucine (Lasic, 1996; Gabizon et al., 2003), which was followed by many other products.

Considering the important role of the PEG layer grafted on the liposomal surface (Barenholz, 2001; Garbuzenko et al., 2005.), its characterization is very critical and is required to be performed for PEGylated liposomal drug delivery systems, as highlighted in a recent review (Jiang et al., 2011). In this review, Jiang et al. also summarized the potential methods which can be used for determination of the PEG-layer thickness, namely measuring the amount of bound water to the PEG layer by 
differential scanning calorimetry and ultrasound velocity measurement (Tirosh et al., 1998), determination of the fixed aqueous layer by measuring the Zeta potential of the liposomes at different ionic strengths (Sadzuka et al., 2002), and describing the interaction of the PEG layer with the surrounding water molecules by NMR spectroscopy (Garcia-Fuentes et al., 2004). All these investigations can reveal the structure of the PEG chains, however the observed quantities in these methods are in indirect connection with it. None of these methods give direct information on the conformation and thickness of the PEG layer in contrast with small-angle X-ray scattering (SAXS) which provides a direct measurement of that.

SAXS is the method of choice for the structural characterization of materials on the nanometer size range, and it is commonly used for the study of liposomes (see e.g. Pabst et al., 2010 and citations therein). Most of these investigations deal with multilamellar systems, which yield quasi-Bragg peaks on the diffraction pattern. The lamellar repeating distance can be obtained from the position of the peak, while the relative intensities of them enable the deduction of the electron density (ED) profile of the bilayer building block (Blaurock and Wothington, 1966). Applying the osmotic stress method in small-angle X-ray scattering, Kenworthy et al. (1995a,b) and Hristova et al. (1995b) studied the structure of bilayers containing PEG-lipids with different molar weights, and related the observed pressure - distance relations to the theoretical models of Alexander, de Gennes and Milner on the conformation of surface grafted polymers (Alexander, 1977; de Gennes, 1980; Milner at al., 1988; Marsh at al., 2003). There exist also full q-range models for the interpretation of the whole SAXS curve of multilamellar liposomes, which serve information also about the interactions between the bilayers (Pabst et al. 2003).

There are less investigations dealing with unilamellar liposomes (or vesicels, ULVs), the scattering pattern of which contains only the 'diffuse scattering' from the uncorrelated bilayers. ULVs are usually prepared by extrusion using a polycarbonate filter with a defined pore size. This method enables to produce diluted samples that - together with fact that the electron density contrast between the water and the bilayer is small - results relatively weak scattering signal in case of SAXS. This is the reason behind that these investigations are usually carried out at synchrotron sources, but state-of-the-art laboratory instruments can also reach that sensitivity (Nadler et al., 2011). SAXS was already applied to describe the influence of extrusion on the average number of bilayers (Jousma et al., 1987), but also to describe the electron density profile of different ULV systems from synthetic (Bouwstra et al., 1993; Hirai et al., 2003; Brzustowicz and Brunger, 2005), and natural origin (Castorph et al, 2010). Small-angle neutron scattering was also applied to study ULVs (Uhriková et al., 2008; Kiselev et al., 2003; Arleth and Vermehren, 2010), which exploits the large scattering length density contrast of protonated lipids and heavy water.

Recently, we applied SAXS to describe the bilayer structure of an SSL system (Varga et al., 2010) based on the Gaussian model of Brzustowicz and Brunger (2005). A different approach was applied by Arleth and Vermehren for the interpretation of SANS data on different SSLs (Arleth and Vermehren, 2010). In this article we present the SAXS analysis of a liposome system with pharmacologically relevant lipid composition with varying PEG-lipid molar ratio and using PEG-lipids with two different PEG molar weights. We also give the comparison of the resulting ED profile with a theoretical prediction on the monomer density of surface grafted polymers (Milner et al., 1988). 


\section{Experimental}

\subsection{Materials and methods}

Synthetic high purity hydrogenated soy lecithine (HSPC) was purchased from NOF Corporation (Japan), 1,2-Distearoyl-sn-Glycero-3-Phosphoethanolamine-N-[Methoxy (Polyethylene glycol)- 2000] (DSPE-PEG2k) and 1,2-Dimyristoyl-sn-Glycero-3-Phosphoethanolamine-N-[Methoxy (Polyethylene glycol)- 1000] (DMPE-PEG1k) were purchased from Avanti Polar Lipids (U.S.A.). Cholesterol was purchased from Sigma (Germany). All the chemicals were used without further purification. Unilamellar liposome samples were prepared by the thin film method. Briefly, the components in the weight ratios of HSPC:DSPE-PEG2k:Cholesterol = 3:1:1, 3:2:1 and 3:0.5:1 (corresponding to the molar ratios of $0.565: 0.053: 0.382,0.537: 0.1: 0.363$, and 0.58:0.027:0.393, respectively) for the samples referred as SSL-1, SSL-2, and SSL-0.5, respectively, and HSPC:DMPE-PEG1k:Cholesterol = 3:0.6:1 (corresponding to the molar ratios of 0.565:0.053:0.382) for the sample referred as SSL-PEG1k were dissolved in chloroform:methanol mixture $(2: 1 \mathrm{v} / \mathrm{v})$. The solvent was then evaporated at $40{ }^{\circ} \mathrm{C}$ and the resulting lipid film was kept in vacuum overnight to remove residual traces of the solvent. 10 $\mathrm{mM}, \mathrm{pH}=7.4$ Tris (Sigma, Germany) buffer solution made of ultrapure water $(18.2 \mathrm{M} \Omega \mathrm{cm})$ was added to the samples to gain a total lipid concentration of $16.2 \mathrm{mg} / \mathrm{ml}$. Ten freeze-thaw cycles by using liquid nitrogen and lukewarm water bath were applied for homogenization. Finally, the samples were extruded ten times through polycarbonate filters (Nucleopore, Whatman Inc.) with $100 \mathrm{~nm}$ pore size using a LIPEX extruder (Northern Lipids Inc., Canada). The extrusion was performed at $60^{\circ} \mathrm{C}$. We chose the above mentioned composition for the SSL-1 system because it is identical to that of the first commercially available liposomal drug, namely the Doxil/Caelyx.

\subsection{SAXS measurements}

SAXS measurements were performed on beamline B1 (Haubold et al., 1989) at HASYLAB at DESY (Hamburg, Germany) and on beamline ID02 (Bösecke, 1992; Narayanan et al., 2001) at ESRF (Grenoble, France). At B1, the samples were filled into quartz capillaries with $1 \mathrm{~mm}$ diameter (Hilgenberg Ltd., Germany). The energy of the incoming X-ray beam was set to $8.6 \mathrm{keV}$, and the 2D scattering patterns were collected with a Pilatus $1 \mathrm{M}$ hybride-pixel detector (Dectris Ltd., Switzerland) with an exposure time of $10 \mathrm{~min}$. At ID02, a flow-through capillary cell with $1.8 \mathrm{~mm}$ diameter was used. The energy of the incoming $\mathrm{X}$-ray beam was set to $12 \mathrm{keV}$ and the $2 \mathrm{D}$ scattering patterns were collected with a FReLON CCD detector with an exposure time of $100 \mathrm{~ms}$. All the measurements were carried out at room temperature. The scattering patterns were corrected for background scattering, and the geometry of the experimental arrangement. The scattering curves were obtained by radial averaging of the patterns and were normalized to the primary beam intensity and corrected for transmission. Finally, the calibration of the curves to absolute units of macroscopic cross section $\left(\mathrm{cm}^{-}\right.$ $\left.{ }^{1}\right)$ was performed by the use of a glassy carbon standard (B1) and water (ID02).

\subsection{DLS measurements}

The particle size distribution of the liposomal systems was determined utilizing dynamic light scattering (DLS) with a Zetasizer Nano ZS (Malvern Instruments, U.K.) instrument. The intensity averaged diameter distribution was characterized by the average value and by the polydispersity index (PDI). 


\subsection{Modell fitting procedures}

In order to describe the electron density (ED) profile along the normal of the phospholipid bilayer, we applied a Gaussian model (Brzustowicz and Brunger 2005; Varga et al., 2010). Briefly, the $E D$ is described by the sum of Gaussian functions, hence the scattered intensity as the function of the scattering variable, $q=\left(\frac{4 \pi}{\lambda}\right) \sin \theta$, where $\theta$ is half the scattering angle and $\lambda$ is the wavelength of the incident X-ray beam, reads

$I(q) \propto\left\langle F(q)^{2}\right\rangle=q^{-2} \sum_{k, k^{\prime}}\left(R_{0}+z_{k}\right)\left(R_{0}+z_{k^{\prime}}\right) \rho_{k} \rho_{k^{\prime}} \sigma_{k} \sigma_{k^{\prime}} \times$

$\exp \left(-q^{2}\left(\sigma_{k}^{2}+\sigma_{k^{\prime}}^{2}\right) / 2\right) \cos \left(q\left(z_{k}-z_{k^{\prime}}\right)\right)$,

where $\rho_{k}, z_{k}$, and $\sigma_{k}$ is the relative weight, position, and width of the $k$ th Gaussian function (Brzustowicz and Brunger, 2004). The average radius of the liposomes is taken into account with $R_{0}$. Previously we have found that the SAXS curve of an SSL system can be adequately described by a so called $2 \mathrm{G}$ model, in which the hydrocarbon chain region is represented by one, while the headgroup region by two Gaussian functions. These two functions describe the phosphate group region and the PEG shell. During the model fitting procedures presented in this paper we applied two different approaches, one corresponding to a symmetric bilayer, i.e. only with three independent functions, and the other which takes into account the asymmetric distribution of the PEG-chains among the inner and outer monolayers, i.e. four functions were independently varied. The headgroup Gaussians were always assumed to be symmetric to the bilayer center. Non-linear least square fitting according to the Levenberg - Marquardt algorithm was performed by using the "Isqcurvefit" routine of Matlab (MathWorks, U.S.A.).

\section{Results and discussion}

The SAXS curves of the sterically stabilized liposome samples containing different concentrations of PEG-lipid (namely SSL-1, SSL-0.5 and SSL-2) are shown in Fig. 1, together with the SAXS curve of the sample containing DMPE-PEG1000 (SSL-PEG1k). The best fitting model functions using Eq. 1 are also shown in Fig. 1. As mentioned previously, we fitted the symmetric and asymmetric models for all the four cases, and the resulting parameters summarized in Table 1 . The radius of the liposomes determined based on DLS measurements are presented in Table 2. The results show that all the four samples contain unimodal distribution of liposomes with average diameter of approximately $100 \mathrm{~nm}$. Since a small deviation in $R_{0}$ causes negligible differences in the calculated curves, $R_{0}=50 \mathrm{~nm}$ was fixed for all the four cases during the model fitting.

The comparison of the two models, i.e. the symmetric and asymmetric ones, for the SAXS curve of the SSL-1 sample, shows that the introduction of asymmetry in the distribution of the PEG-chains causes small, but systematic improvement in the fit. Previously we have observed that the asymmetric model results an increase in the intensity at the local minima of the scattering curves in the q-range of $0.35-0.4 \mathrm{~nm}^{-1}$ (Varga et al., 2010). According to the plot of residuals versus q for the two models, significant difference can be seen in the above mentioned q-range, underlining the asymmetric distribution of the PEG-lipids among the two monolayers. The ED profiles of the bilayer 
for the SSL-1 sample according to the two fits are shown in Fig. 2. The contribution of the headgroup, the hydrocarbon, and the PEG-regions are also displayed in the figure.

The SAXS curves for the systems SSL-2, SSL-0.5 and SSL-PEG1k shown in Fig. 1 indicate that the asymmetric model gives a definitely better fit for SSL-2, where the mole fraction of the PEG-lipids is doubled in comparison to the sample SSL-1. On the other hand, there is no significant difference in the two models, if the mole fraction of the PEG-lipid is halved (SSL-0.5) or a PEG-lipid with halved molar weight of the PEG group is used (SSL-PEG1k). The latter is also reflected in the errors of the parameters corresponding to the best fits in these cases, namely the increased errors for the parameters of the PEG-chains in the asymmetric model show that this model is overparametrized for the scattering curves of these samples. In summary, the asymmetric model applies for the SSL-1 and SSL-2 samples, while the symmetric model is acceptable for the SSL-0.5 and SSL-PEG1k samples. The corresponding ED profiles are shown in Fig. 3.

The results from the fitting of the SAXS curves can be compared to the theoretical predictions. There are two different cases for the conformation of the lipopolymers grafted on the bilayer surface depending on the relationship between the Flory radius of the polymer group (defined as $R_{F}=$ $N^{3 / 5} a$, where $\mathrm{N}$ is the number of monomers and $a$ is the size of the monomer unit) and the grafting density of them. The so called "mushroom" conformation is formed when the polymer coils do not overlap, while in the opposite case, the interaction between the neighboring polymer coils leads to the formation of the "brush" structure. These structures were first described by the Alexander - de Gennes scaling theory (Alexander, 1977; de Gennes, 1980), while a different, mean-field treatment was proposed by Milner et al. (1988). According to these theories, the equilibrium length of the polymer chain in the brush conformation can be expressed as

$L^{M F}=N a^{5 / 3} D^{-2 / 3}$,

with $\mathrm{D}$ being the distance between the grafting points defined as $D^{2}=A_{l} / X_{p}$, where $A_{l}$ is the area per lipid and $X_{p}$ is the mole fraction of the PEG-lipid (Marsh et al., 2003).

In order to calculate $\mathrm{L}^{\mathrm{MF}}$ and $\mathrm{R}_{\mathrm{F}}$, the size of the monomer and the grafting density of the PEG-chains have to be estimated. The reported size of the oxyethylene unit is $0.39 \mathrm{~nm}$ (Evans et al., 1996), but values of $0.35 \mathrm{~nm}$ from theoretical predictions (Hristova and Needham, 1995b) and $0.43 \mathrm{~nm}$ from adhesion measurements (Evans et al., 1996) also appear in the literature. The grafting density, which can be calculated from the mole fraction of the PEG-lipids and from the area per lipid $\left(A_{L}\right)$, also relies on assumption of the latter. Using $\mathrm{N}=45$ (for PEG2000), $a=0.39 \mathrm{~nm}$ and $A_{L}=0.7 \mathrm{~nm}^{2}$ one obtains $\mathrm{L}^{\mathrm{MF}}$ $=4.0 \mathrm{~nm}$, but using the same area per lipid with $\mathrm{a}=0.43 \mathrm{~nm}$ gives $\mathrm{L}^{\mathrm{MF}}=4.7 \mathrm{~nm}$ for the grafting density corresponding to the SSL- 1 sample. The values of $a=0.39 \mathrm{~nm}$ and $A_{L}=0.5 \mathrm{~nm}^{2}$ result $L^{\mathrm{MF}}=4.4 \mathrm{~nm}$. In order to obtain the monomer density as a function of the distance from the surface (z), Milner et al. (1988) have developed a self-consistent mean-field theory for polymer brushes. As a result, they obtained a quadratic dependence:

$\Phi(z) \sim\left(L^{M F}\right)^{2}-z^{2}$

which was in accordance with previous Monte Carlo simulations. In Fig. 4 we compare the ED contribution of the PEG-chains according to the Gaussian model function for the outer leaflet from the fitting of the SAXS data of the SSL-1 sample with the theoretical segment density distribution 
from Milner et al. using the above mentioned $L^{M F}$ values. The grafting plane was defined as $z_{G P}=z_{H}+2 \sqrt{2 \ln 10} * \sigma_{H}$, where $z_{H}$ and $\sigma_{H}$ are the position and the width of the Gaussian function representing the headgroup region. The latter definition takes into account the distance between the first monomer of the PEG chain and the phosphate group of the lipids (maxima of the headgroup Gaussian). The two distributions are comparable, apart from the different decay of the functions at larger $z$ values. The theoretical profile matches to that obtained from the SAXS fit with the value of $\mathrm{a}=0.43 \mathrm{~nm}$ that is slightly larger than the theoretical size of the monomer in accordance with previous adhesion measurements on PEG grafted liposomes (Evans et al., 1996).

Based on these results, we propose to use $L=\left(z_{P E G}-z_{G P}\right)+2 * \sigma_{P E G}$, with $z_{\text {PEG }}$ and $\sigma_{P E G}$ being the position and the width of the Gaussian function representing the PEG chains, for the characterization of the PEG layer thickness in the framework of the Gaussian model. This assumption leads to the values summarized in Table 3. In the case of SSL-0.5 and SSL-2 samples, the structures of the polymer layers are according to the "mushroom" and "brush" configurations, respectively, while the SSL-1 sample represents an intermediate between these two cases according to Garbuzenko et al. (2005). Using a $=0.43 \mathrm{~nm}$ for the size of the monomer gives $4.2 \mathrm{~nm}$ and $2.6 \mathrm{~nm}$ for the Flory-radii of PEG2000, and PEG1000, respectively, which agrees well with the $4.1 \pm 0.1 \mathrm{~nm}$ and $2.7 \pm 0.1 \mathrm{~nm}$ for the average thicknesses of the PEG layers for the SSL-0.5 and SSL-PEG1k samples obtained from the SAXS fit. The value for the SSL-PEG1k sample implies that this system is also in the "mushroom" conformation in accordance with the consideration of its Flory-radius and the grafting density of this sample. For the SSL-1 sample, the asymmetric model applies, which results also different thicknesses of the PEG layers among the inner and outer leaflets. The obtained values for the latter are close to the theoretical value of $4.7 \mathrm{~nm}$ using $0.7 \mathrm{~nm}^{2}$ for the area per lipid, and the above mentioned value for the size of the monomer. The largest difference between the values obtained from the SAXS fit and from the mean-field theory can be seen in the case of the SSL-2 sample. In this case, the meanfield approximation results $5.8 \mathrm{~nm}$ while the measured values are $4.8 \pm 0.2$ and $5.1 \pm 0.1 \mathrm{~nm}$ for the inner and outer leaflets, respectively. There are several reasons that can account for this deviation: Either the validity of the mean-field model and the parameters used can be questionable, or the Gaussian model in the SAXS fit or the applied procedure to calculate the PEG-layer thickness from the ED profile can be inadequate for this composition. According to Garbuzenko et al. (2005) the PEGlipid molar ratio of the SSL-2 sample is far from the bilayer to micelle transition (which occurs at 30 mol\% PEG-lipid), but the presence of PEG-lipid micelles can also account for the later observation. On the other hand, significant amount of micelles would also contribute to the SAXS curve of the system in the used q-range.

Independently from the PEG-layer thickness, we calculated the integrated electron densities of the PEG-layers for the SSL-1 and SSL-2 samples in order to quantify the partitioning of the PEG-lipids among the inner and outer monolayers. We used the ED profiles from the fits, and performed the integration with the limits defined by the grafting planes, and taking into account the spherical correction, i.e. integrated $r^{2} \rho(r)$. We obtained 0.33:0.67 for the ratio of the inner/outer PEG-chains for the SSL-1 sample and 0.25:0.75 for the SSL-2 sample, which is in semi-quantitative agreement with the prediction of 0.4:0.6 from theoretical predictions (Rovira-Bru et al., 2002). 


\section{Conclusions}

The SAXS analysis of the thickness of the PEG-layer of four sterically stabilized liposome samples is presented. By changing the molar ratio and type of the PEG-lipids in the liposome composition, we observed systematic changes in the scattering curves. Applying a Gaussian model, we identified the contribution of the PEG-layers to the total electron density profile and obtained a good agreement with the theoretical predictions of the self-consistent mean-field calculation on the structure of surface grafted polymers. We got asymmetric distribution of the PEG-lipids for the lipid composition identical to Doxil, the first approved liposomal drug, and symmetric for the same composition but containing a PEG-lipid with halved molar weight of the PEG group. The distribution of the PEG-lipids is also asymmetric for the sample with doubled, and symmetric with halved molar ratio of the PEGlipid.

\section{Acknowledgement}

This work was supported by the Hungarian Scientific Research Fund (OTKA, Hungary) and the National Innovation Office ( $\mathrm{NIH}$, Hungary) under grant agreement CNK-81056. The research leading to these results has received funding from the European Community's Seventh Framework Programme (FP7/2007-2013) under grant agreement $n^{\circ} 226716$ at DESY/HASYLAB. We are grateful to the European Synchrotron Radiation Facility for provision of beam time at ID02 (proposal no. SC3269).

\section{References}

Alexander, S., 1977. Adsorption of chain molecules with polar head. A scaling description. J. de Phys. $38,983-987$.

Arleth, L., Vermehren, C., 2010. An analytical model for the small-angle scattering of polyethylene glycol modified liposomes. J. Appl. Cryst. 43, 1084-1091.

Bangham, A. D., 1995. Surrogate cells or Trojan horses. BioEssays 17, 1081-1088.

Barenholz, Y., 2001. Liposome application: problems and prospects. Curr. Opin. Coll. Interface. Sci. 6, 66-77.

Blaurock A. E., Worthington, C. R., 1966. Treatment of low angle x-ray data from planar and oncentric multilayered structures. Biophys. J. 6, 305-312.

Bouwstra, J.A., Gooris, G.S., Bras, W., Talsma H., 1993. Small angle X-ray scattering: possibilities and limitations in characterization of vesicles. Chem. Phys. Lipids 64, 83-98.

Bösecke, P., 1992. The high-flux beamline at the ESRF. Rev. Sci. Instrum. 63, 438-441. 
Brzustowicz, M. R., Brunger, A. T., 2005. X-ray scattering from unilamellar lipid vesicles. J. Appl. Cryst. 38, 126-131.

Castorph, S., Riedel, D., Arleth, L., Sztucki, M., Jahn, R., Holt, M., Salditt, T., 2010. Structure parameters of synaptic vesicles quantified by small-angle X-Ray Scattering. Biophys. J. 98, 1200-1208

Evans E., Klingenber, D. J., Rawicz, W., Szoka, F., 1996. Interactions between polymer grafted membranes in concentrated solutions of free polymer. Langmuir 12, 3031-3037.

Gabizon, A., Sheemda, H., Barenholz, Y., 2003. Pharmacokinetics of pegylated liposome doxorubicin: review of animal and human studies. Clin Pharmcokinet 42, 419-436.

Garbuzenko, O., Barenholz, Y., Priev, A., 2005. Effect of grafted PEG on liposome size and on compressibility and packing of lipid bilayer. Chem. Phys. Lipids 135, 117-129.

Garcia-Fuentes, M., Torres, D., Martín-Pastor, M., Alonso, M. J., 2004. Application of NMR spectroscopy to the characterization of PEG-stabilized lipid nanoparticles. Langmuir 20, 8839-8845.

de Gennes, P. G., 1980. Conformations of Polymers Attached to an Interface. Macromolecules 13, 1069-1075.

Haubold, H.-G., Gruenhagen, K., Wagener, M., Jungbluth, H., Heer, H., Pfeil, A., Rongen, H., Brandenberg, G., Moeller, R., Matzerath, J., Hiller, P., Halling, H., 1989. JUSIFA-A new user-dedicated ASAXS beamline for materials science. Rev. Sci. Instrum. 60, 1943 - 1946.

Hirai, M., Iwase, H., Hayakawa, T., Koizumi M., Takahashi, H., 2003. Determination of asymmetric structure of ganglioside-DPPC mixed vesicle using SANS, SAXS, and DLS. Biophys. J. 85, 1600-1610.

Hristova, K., Kenworthy, A., McIntosh, T. J., 1995a. Effect of Bilayer Composition on the Phase Behavior of Liposomal Suspensions Containing Poly(ethylene glycol)-lipids. Macromolecules 28, 76937699.

Hristova, K., Needham, D., 1995b. Phase behavior of a lipid/polymer-lipid mixture in aqueous medium. Macromolecules 28, 991-1002.

Jiang, W., Lionberger, R., Yu, L.X., 2011. In vitro and in vivo characterizations of PEGylated liposomal doxorubicin. Bioanalysis 3(3), 333-344.

Jousma, H., Talsma, H., Spies, F., Joosten, J.G.H., Junginger, H.E., Crommelin, D.J.A., 1987. Characterization of liposomes. The influence of extrusion of multilamellar vesicles through polycarbonate membranes on particle size, particle size distribution and number of bilayers. Int. J. Pharm. 35, 263-274.

Kenworthy, A. K., Hristova, K., Needham, D., McIntosh, T.J., 1995a. Range and Magnitude of the Steric Pressure Between Bilayers Containing Phospholipids with Covalently Attached Poly(ethylene glycol). Biophys. J. 68, 1921-1936.

Kenworthy, A. K., Simon, S. A., McIntosh, T. J., 1995b. Structure and Phase Behavior of Lipid Suspensions Containing Phospholipids with Covalently Attached Poly(ethylene glycol). Biophys. J. 68, 1903-1920. 
Kiselev, M.A., Wartewig, S., Janich, M., Lesieur, P., Kiselev, A.M., Ollivon, M., Neubert, R., 2003. Does sucrose influence the properties of DMPC vesicles? Chem. Phys. Lipids 123, 31-44.

Lasic, D. D., 1996. Doxorubicin in sterically stabilized liposomes. Nature 380, 561-562

Marsh, D., Bartucci, R., Sportelli, L., 2003. Lipid membranes with grafted polymers: physicochemical aspects. Biochimica et Biophysica Acta 1615, 33-59.

Milner, S.T., Witten, T. A., Cates, M. E., 1988. Theory of the Grafted Polymer Brush. Macromolecules 21, 2610-2619.

Nadler, M., Steiner, A., Dvir, T., Szekely, O., Szekely, P., Ginsburg, A., Asor,R., Resh, R., Tamburu,C., Peres, M., Raviv, U., 2011. Following the structural changes during zinc-induced crystallization of charged membranes using time-resolved solution X-ray scattering. Soft Matter 7, 1512-1523.

Narayanan, T., Diat, O., Bösecke, P., 2001. SAXS and USAXS on the high brilliance beamline at the ESRF. Nucl. Instrum. Methods Phys. Res. A, 467-468, 1005-1009.

Pabst, G., Kucerka, N., Nieh, M.-P., Rheinstädter, M.C., Katsaras, J., 2010. Applications of neutron and X-ray scattering to the study of biologically relevant model membranes. Chem. Phys. Lipids 163, 460-479.

Pabst, G., Koschuch, R., Pozo-Navas, B., Rappolt, M., Lohner, K., Laggner, P., 2003. Structural analysis of weakly ordered membrane stacks. J. Appl. Cryst. 36, 1378-1388.

Rovira-Bru, M., Thompson, D. H., Szleifer, I., 2002. Size and Structure of Spontaneously Forming liposomes in Lipid/PEG-Lipid Mixtures. Biophys. J. 83, 2419-2439.

Sadzuka, Y., Nakade, A., Hirama, R., Miyagishima, A., Nozawa, Y., Hirota, S., Sonobe, T., 2002. Effects of mixed polyethyleneglycol modification on fixed aqueous layer thickness and antitumor activity of doxorubicin containing liposome. Int. J. Pharm. Sci. 238, 171-180.

Tirosh, O., Barenholz, Y., Katzhendler, J., Priev, A., 1998. Hydration of polyethylene glycol-grafted liposomes. Biophys. J. 74, 1371-1379.

Uhríková, D., Kucerka, N., Teixeira, J., Gordeliy, V., Balgavy, P. 2008. Structural changes in dipalmitoylphosphatidylcholine bilayer promoted by $\mathrm{Ca}^{2+}$ ions: a small-angle neutron scattering study. Chem. Phys. Lipids 155, 80-89.

Varga, Z., Berényi, Sz., Szokol, B., Őrfi, L., Kéri, Gy., Peták, I., Hoell, A., Bóta, A., 2010. A Closer Look at the Structure of Sterically Stabilized Liposomes: A Small-Angle X-ray Scattering Study. J. Phys. Chem. B. $114,6850-6854$. 

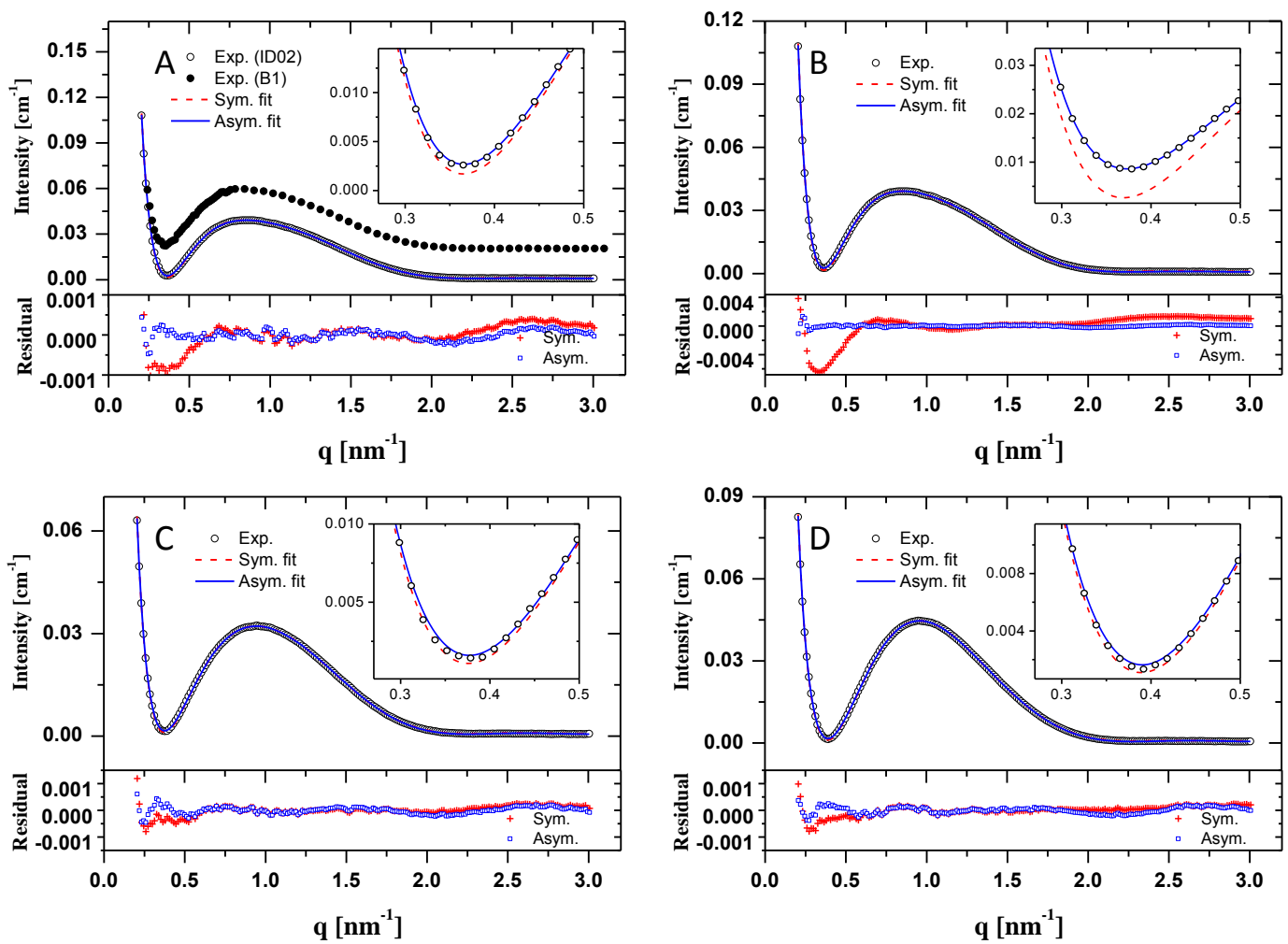

Fig. 1 Measured SAXS curves corresponding to the liposome composition in weight ratio of HSPC:DSPE-PEG2k:Cholesterol 3:1:1 (SSL-1: A), 3:2:1 (SSL-2: B), 3:0.5:1 (SSL-0.5: C) and HSPC:DMPEPEG1k:Cholesterol 3:0.6:1 (SSL-PEG1k: D). The best model fits using the symmetric (dashed red line) and asymmetric (solid blue line) models are also shown in the figure. The insets show the q-region of $0.3-0.5 \mathrm{~nm}^{-1}$, where there are a significant differences between the two models for the SSL- 1 and SSL-2 samples ( $A$ and $B$, respectively). The reproducibility of the measurements is demonstrated in $A$ (SSL-1 sample), where the measured curves from the B1 and ID02 beamlines are shown (the curve from B1 is shifted for better visibility).

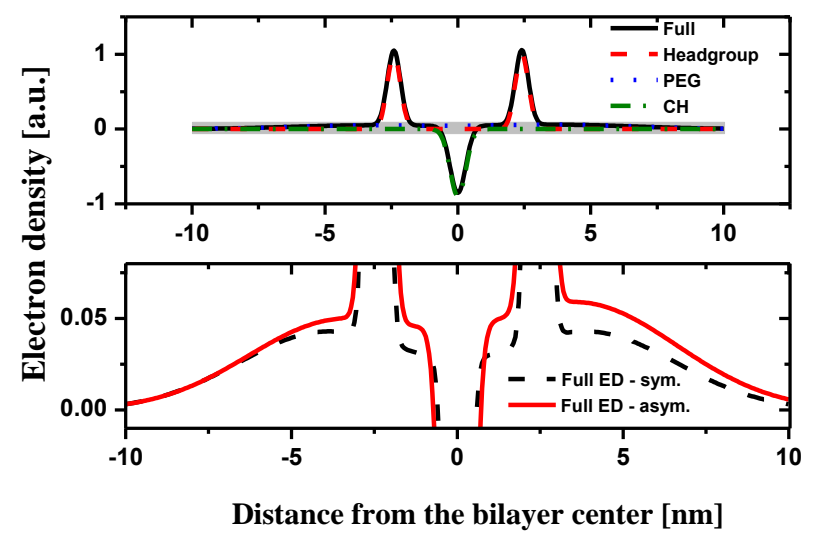


Fig. 2 The electron density profile of the bilayer corresponding to the SSL-1 sample. The contribution of the separate Gaussian functions is presented in the upper figure. The distribution of the PEGchains according to the symmetric (dashed black line) and asymmetric (full red line) models are shown in the lower figure.

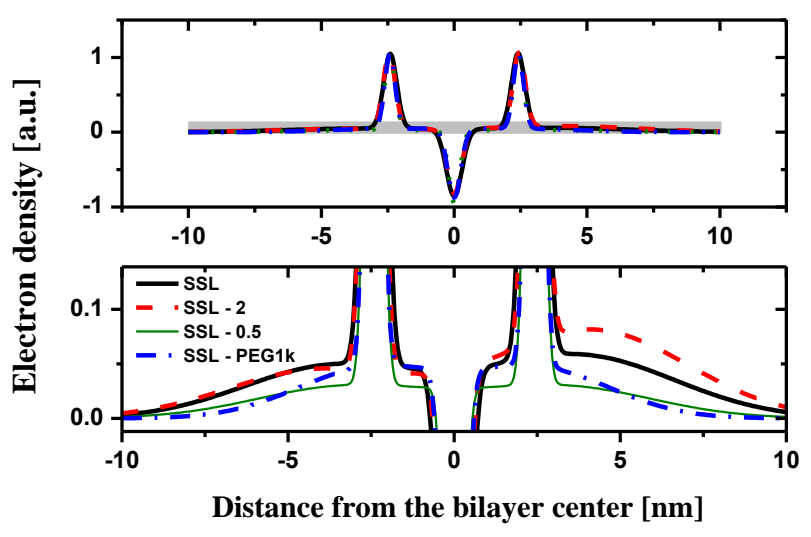

Fig. 3 The electron density profiles of the four sterically stabilized liposome samples. The profiles correspond to the asymmetric model in the case of the SSL-1 and SSL-2 samples, and to the symmetric model in the case of the SSL-0.5 and SSL-PEG1k samples.

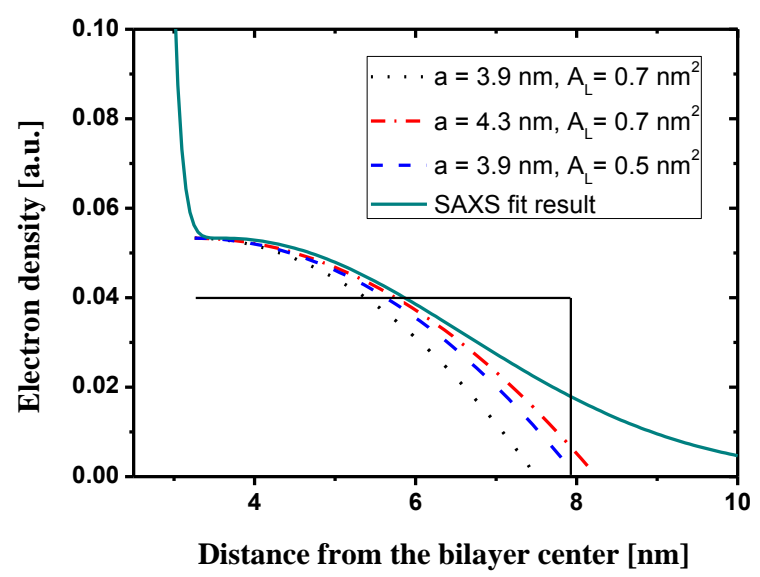

Fig. 4 The contribution of the PEG layers to the total electron density in the case of the SSL-1 sample (outer monolayer). The profile from the SAXS fit is compared to the theoretical monomer density according to the mean-field approximation with different parameters. The step function with $\mathrm{L}^{\mathrm{MF}}=4.7$ $\mathrm{nm}$ is also indicated in the figure. 


\begin{tabular}{|c|c|c|c|c|}
\hline \multirow[b]{2}{*}{ Parameter } & \multicolumn{2}{|c|}{ SSL } & \multicolumn{2}{|c|}{ SSL-2 } \\
\hline & $\begin{array}{l}\text { Symmetric } \\
\text { model }\end{array}$ & $\begin{array}{c}\text { Asymmetric } \\
\text { model }\end{array}$ & $\begin{array}{l}\text { Symmetric } \\
\text { model }\end{array}$ & $\begin{array}{l}\text { Asymmetric } \\
\text { model }\end{array}$ \\
\hline$\sigma_{\mathrm{PEG}}(\mathrm{in})[\mathrm{nm}]$ & $1.88 \pm 0.01$ & $1.93 \pm 0.09$ & $1.69 \pm 0.02$ & $1.98 \pm 0.07$ \\
\hline$\sigma_{\mathrm{PEG}}$ (out) [nm] & $=\sigma_{\mathrm{PEG}}$ (in) & $2.07 \pm 0.04$ & $\sigma_{P E G}($ in) & $2.04 \pm 0.02$ \\
\hline$\sigma_{H}[\mathrm{~nm}]$ & $0.15 \pm 0.02$ & $0.18 \pm 0.01$ & $0.17 \pm 0.05$ & $0.16 \pm 0.01$ \\
\hline$\sigma_{\mathrm{CH}}[\mathrm{nm}]$ & $0.22 \pm 0.01$ & $0.30 \pm 0.01$ & $0.21 \pm 0.07$ & $0.26 \pm 0.01$ \\
\hline $\mathrm{Z}_{\mathrm{PEG}}$ (in) $[\mathrm{nm}]$ & $3.96 \pm 0.02$ & $3.65 \pm 0.12$ & $4.54 \pm 0.04$ & $4.02 \pm 0.15$ \\
\hline $\mathrm{Z}_{\mathrm{PEG}}$ (out) $[\mathrm{nm}]$ & $=z_{P E G}$ (in) & $3.72 \pm 0.05$ & $=Z_{P E G}$ (in) & $4.15 \pm 0.05$ \\
\hline $\mathrm{z}_{\mathrm{H}}[\mathrm{nm}]$ & $2.428 \pm 0.003$ & $2.408 \pm 0.002$ & $2.48 \pm 0.01$ & $2.446 \pm 0.002$ \\
\hline$\rho_{\mathrm{PEG}}$ (in) & $0.042 \pm 0.004$ & $0.047 \pm 0.003$ & $0.06 \pm 0.02$ & $0.044 \pm 0.003$ \\
\hline$\rho_{\text {PEG }}$ (out) & $=\rho_{P E G}$ (in) & $0.058 \pm 0.002$ & $=\rho_{\mathrm{PEG}}$ (in) & $0.081 \pm 0.006$ \\
\hline$\rho_{\mathrm{CH}}$ & $-0.92 \pm 0.05$ & $-0.90 \pm 0.04$ & $-0.93 \pm 0.09$ & $-0.87 \pm 0.05$ \\
\hline
\end{tabular}

\begin{tabular}{|c|c|c|c|c|}
\hline & \multicolumn{2}{|c|}{ SSL-0.5 } & \multicolumn{2}{|c|}{ SSL-PEG1000 } \\
\hline Parameter & $\begin{array}{l}\text { Symmetric } \\
\text { model }\end{array}$ & $\begin{array}{c}\text { Asymmetric } \\
\text { model }\end{array}$ & $\begin{array}{l}\text { Symmetric } \\
\text { model }\end{array}$ & $\begin{array}{c}\text { Asymmetric } \\
\text { model }\end{array}$ \\
\hline$\sigma_{\mathrm{PEG}}(\mathrm{in})[\mathrm{nm}]$ & $1.91 \pm 0.01$ & $1.82 \pm 0.94$ & $1.55 \pm 0.01$ & $1.09 \pm 0.55$ \\
\hline$\sigma_{\mathrm{PEG}}$ (out) [nm] & $=\sigma_{\mathrm{PEG}}$ (in) & $1.81 \pm 0.54$ & $\sigma_{\mathrm{PEG}}$ (in) & $1.42 \pm 0.10$ \\
\hline$\sigma_{\mathrm{H}}[\mathrm{nm}]$ & $0.14 \pm 0.02$ & $0.18 \pm 0.02$ & $0.14 \pm 0.02$ & $0.18 \pm 0.13$ \\
\hline$\sigma_{\mathrm{CH}}[\mathrm{nm}]$ & $0.21 \pm 0.01$ & $0.29 \pm 0.01$ & $0.24 \pm 0.01$ & $0.28 \pm 0.03$ \\
\hline $\mathrm{Z}_{\mathrm{PEG}}$ (in) $[\mathrm{nm}]$ & $3.27 \pm 0.04$ & $3.38 \pm 2.27$ & $2.56 \pm 0.04$ & $3.20 \pm 1.15$ \\
\hline $\mathrm{Z}_{\mathrm{PEG}}$ (out) $[\mathrm{nm}]$ & $=\mathrm{Z}_{\mathrm{PEG}}$ (in) & $3.41 \pm 1.37$ & $=Z_{P E G}$ (in) & $3.07 \pm 0.66$ \\
\hline $\mathrm{z}_{\mathrm{H}}[\mathrm{nm}]$ & $2.415 \pm 0.003$ & $2.387 \pm 0.003$ & $2.420 \pm 0.003$ & $2.37 \pm 0.02$ \\
\hline$\rho_{\text {PEG }}$ (in) & $0.029 \pm 0.003$ & $0.030 \pm 0.002$ & $0.045 \pm 0.004$ & $0.05 \pm 0.01$ \\
\hline$\rho_{\text {PEG }}$ (out) & $=\rho_{P E G}$ (in) & $0.041 \pm 0.003$ & $=\rho_{\mathrm{PEG}}$ (in) & $0.049 \pm 0.004$ \\
\hline$\rho_{\mathrm{CH}}$ & $-0.97 \pm 0.06$ & $-0.89 \pm 0.06$ & $-0.93 \pm 0.09$ & $-0.91 \pm 0.53$ \\
\hline
\end{tabular}

Table 1 The parameters obtained from the least squares fits of the symmetric and asymmetric models for the four investigated samples. $\sigma_{\mathrm{PEG}}, \sigma_{\mathrm{H}}, \sigma_{\mathrm{CH}}$ denote the width of the Gaussian functions describing the PEG, the headgroup, and the hydrocarbon chain regions, respectively. $z_{P E G}, z_{H}$ are the positions of the Gaussian functions for the PEG, and the headgroup regions, while $\rho_{\text {PEG }}$ and $\rho_{C H}$ are the relative weights of the functions for the PEG and hydrocarbon chain regions, respectively. The asymmetric model gives better fit for the SSL-1 and SSL-2 samples, while the symmetric model applies for the SSL-0.5 and SSL-PEG1k samples. The parameters of these cases are highlighted by bold letters.

\begin{tabular}{|c|c|c|}
\hline Sample & $D[n m]$ & PDI \\
\hline SSL - 1 & 105.7 & 0.067 \\
\hline SSL - 0.5 & 110.3 & 0.082 \\
\hline SSL - 2 & 101.5 & 0.085 \\
\hline SSL - PEG1k & 101.7 & 0.071 \\
\hline
\end{tabular}

Table 2 The average diameters (D) and the polydispersity indexes (PDI) of the investigated samples based on DLS measurements. 


\begin{tabular}{|l|c|}
\hline Sample & $\begin{array}{l}\text { Thickness of the } \\
\text { PEG layer [nm] }\end{array}$ \\
\hline SSL - 1 (in) & $4.4 \pm 0.2$ \\
\hline SSL - 1 (out) & $4.7 \pm 0.1$ \\
\hline SSL - 0.5 & $4.1 \pm 0.1$ \\
\hline SSL - 2 (in) & $4.8 \pm 0.2$ \\
\hline SSL - 2 (out) & $5.1 \pm 0.1$ \\
\hline SSL - PEG1k & $2.7 \pm 0.1$ \\
\hline
\end{tabular}

Table 3 The calculated thicknesses of the PEG layers for the four investigated samples based on the electron density profiles determined from the SAXS fits, using the approximation mentioned in the text. 